\title{
Lacrimal Gland Involvement in Lymphomatoid Granulomatosis and Review of the Literature
}

\author{
Thanuja Gopal Pradeep, ${ }^{1,2}$ Paul Cannon, ${ }^{1}$ Thomas Dodd, ${ }^{3}$ and Dinesh Selva ${ }^{1}$ \\ ${ }^{1}$ South Australian Institute of Ophthalmology and Discipline of Ophthalmology and Visual Sciences, University of Adelaide, \\ Adelaide, SA 5000, Australia \\ ${ }^{2}$ Department of Ophthalmology and Visual Sciences, Royal Adelaide Hospital, North Terrace, Adelaide, SA 5000, Australia \\ ${ }^{3}$ Institute of Medical and Veterinary Sciences, University of Adelaide, Adelaide, SA 5000, Australia
}

Correspondence should be addressed to Thanuja Gopal Pradeep, thanugopal@yahoo.co.in

Received 8 June 2010; Accepted 3 August 2010

Academic Editor: Denis Wakefield

Copyright (C) 2010 Thanuja Gopal Pradeep et al. This is an open access article distributed under the Creative Commons Attribution License, which permits unrestricted use, distribution, and reproduction in any medium, provided the original work is properly cited.

\begin{abstract}
Objective. To describe the clinicoradiological and histopathological findings in a case of lacrimal gland enlargement secondary to lymphomatoid granulomatosis (LG) and to review the literature. Design. Case report and systematic literature review. Methods. A 75-year-old woman presented with right ptosis. Computerised tomography showed lacrimal gland enlargement, and biopsy done was inconclusive. She subsequently developed pulmonary symptoms and underwent transbronchial biopsy that was diagnosed as LG. Pub Med and OVID databases were searched using the term "orbit/eye involvement in lymphomatoid granulomatosis". Articles that predated the databases were gathered from current references. Results. The patient underwent lacrimal gland biopsy which revealed necrotic and inflamed tissue with no further categorisation but transbronchial biopsy helped in establishing the diagnosis of LG. On initiation of prednisolone and cyclophosphamide, her orbital lesion resolved but the patient died following massive pulmonary hemorrhage within a month of diagnosis. Conclusion. Ophthalmic involvement in LG is very rare. Varied presentations are due to central nervous system involvement, vasculitis, or infiltration of ocular or orbital structures. LG is an angiocentric and angiodestructive granulomatous disorder and can involve any tissue, thus accounting for the variable presentations reported in literature.
\end{abstract}

\section{Introduction}

LG is an Epstein-Barr virus- (EBV-) associated angiocentric B-cell lymphoproliferative process. The lung is the most commonly involved organ, but skin, central nervous system, kidney, and gastrointestinal tract can also be involved. There are few reports of ophthalmic involvement in LG. We report a patient with LG who presented with dacryoadenitis and review the literature on the ophthalmic manifestations of this condition.

\section{Case Report}

A 75-year-old woman developed a painless acute right ptosis associated with double vision and vertigo. Examination revealed inferomedial globe displacement and an S-shaped ptosis (Figure 1). Computerized tomography (CT) revealed an enlarged right lacrimal gland with areas of central hypodensity (Figure 2). The medical history included Sjogren's syndrome, abdominal aortic aneurysm, and colonic adenocarcinoma 2 years previously that was treated surgically. The lacrimal gland was biopsied through a superior lid crease approach. Histopathology was inconclusive showing only necrotic and inflamed tissue with granulomatous inflammation (Figure 3). A further biopsy was declined by the patient.

She subsequently developed fever, malaise, and cough. A $\mathrm{CT}$ of the chest showed a necrotic mass involving her left upper lobe and hilar vessels with a suspicious right lung lesion. Hilar and mediastinal lymphadenopathy was noted. CT abdomen revealed multiple hepatic lesions. She underwent bronchial biopsy and the histopathology demonstrated 


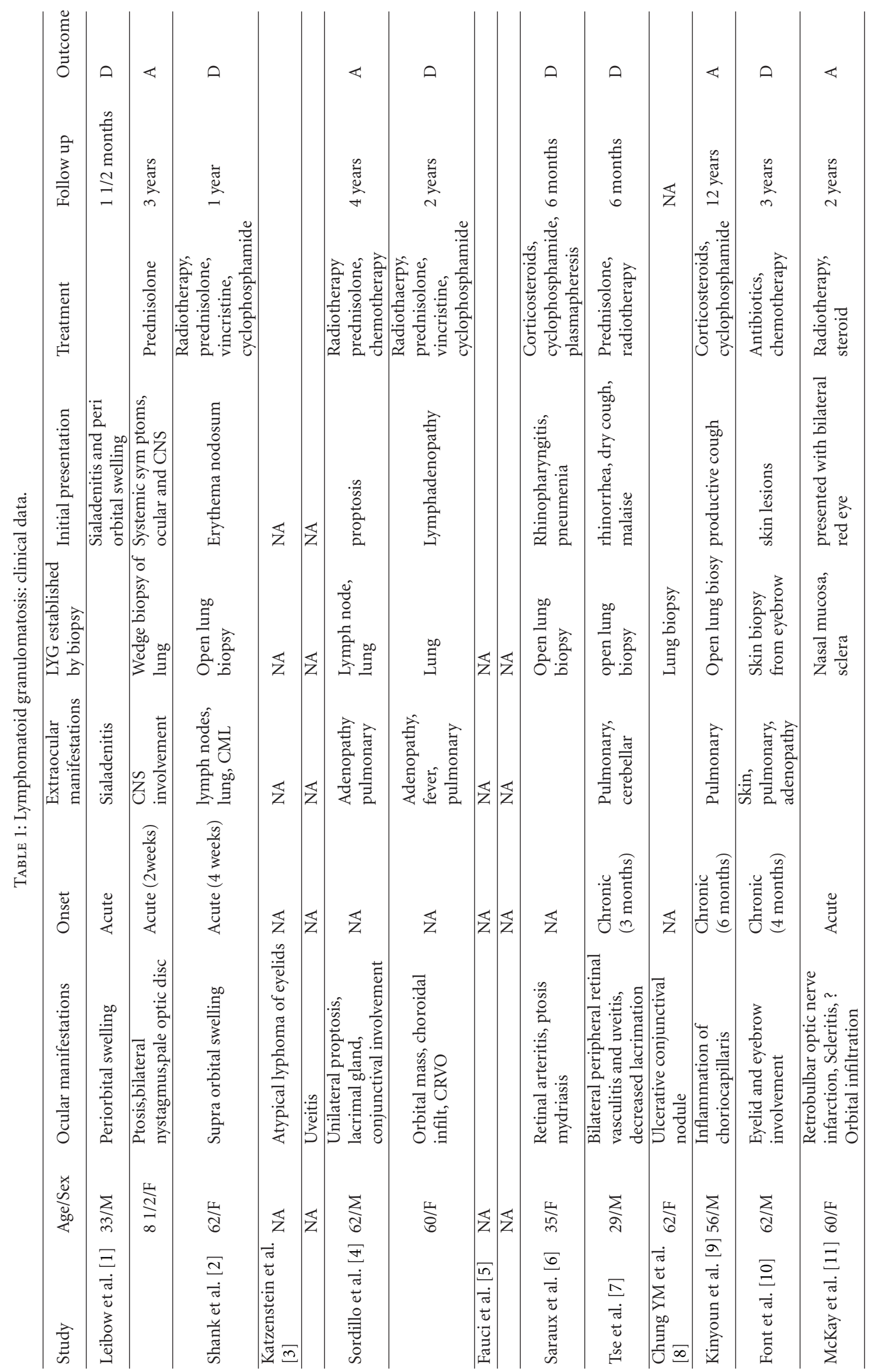




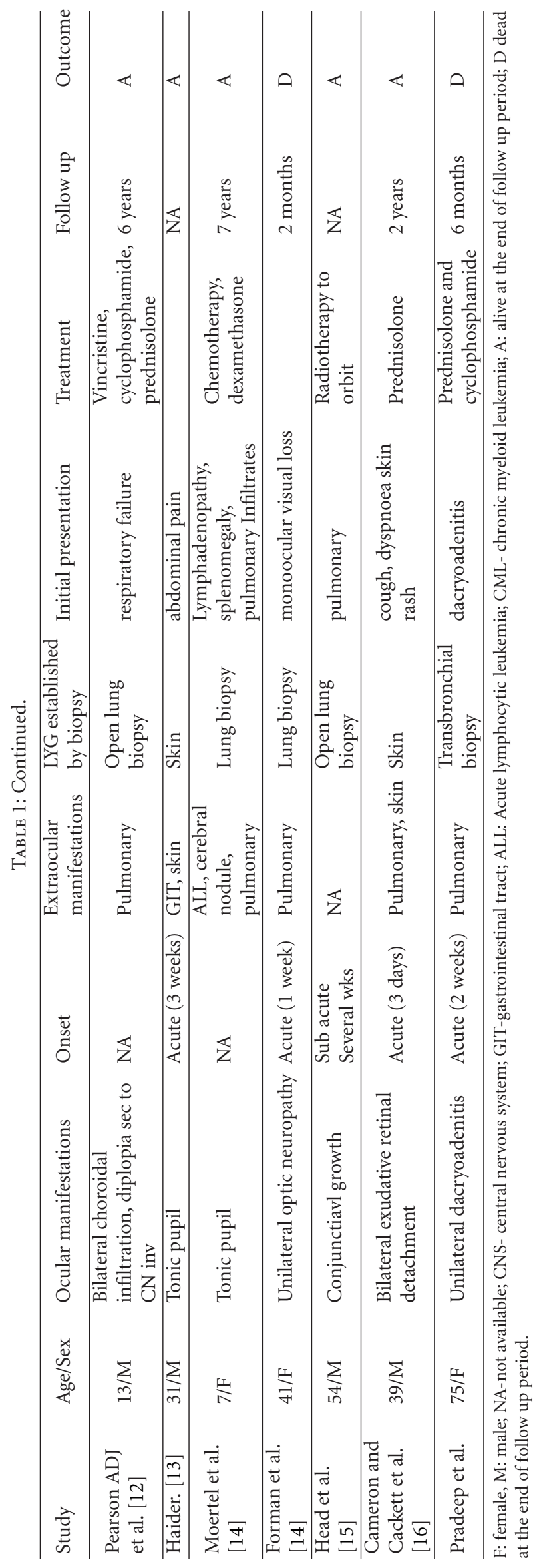




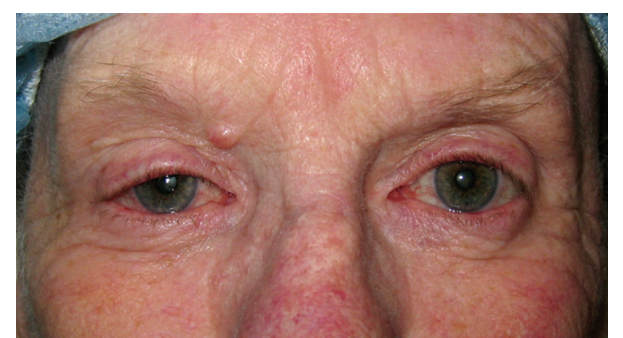

FIGURE 1: Clinical photograph showing inferomedial displacement of the globe.

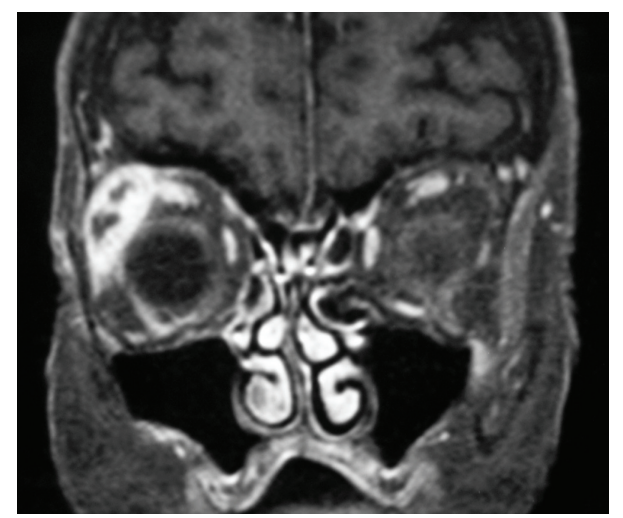

FIGURE 2: T1 weighted contrast enhanced MRI scan coronal sections through the orbits showing contrast enhancing lacrimal gland lesions with central hypodense areas.

florid necrotizing inflammation with palisading granulomas and a probable diagnosis of Wegner's granulomatosis was made. Her blood investigations showed elevated CRP levels $(150 \mathrm{mg} / \mathrm{l})$ and anemia $(\mathrm{Hb}-10 \mathrm{~g} / \mathrm{dl})$. ANCA was negative and CEA levels were normal $(2 \mu \mathrm{g} / \mathrm{L})$. The patient was started on Prednisolone $25 \mathrm{mg} /$ day and Cyclophosphamide $100 \mathrm{mg} /$ day.

She was readmitted 1 month later with further deterioration of her systemic condition. CT of the orbits were normal with complete resolution of the lacrimal gland enlargement. However, her CT of the chest revealed development of left lower lobe parenchymal infiltrates and resolution of liver lesions when compared with the previous study. She underwent a repeat bronchial biopsy and the histopathology showed a polymorphous atypical lymphoid infiltrate of CD20 positive $\mathrm{B}$ cells with positive labelling for EBV admixed with $\mathrm{CD} 3$ positive $\mathrm{T}$ cells. The lesion displayed focal necrosis and focal angiocentricity and a diagnosis of LG with areas of high grade involvement was made (Figure 4). Microbiology yielded Staphylococcus aureus. She was treated with antibiotics and steroids and cyclophosphamide. She underwent bone marrow biopsy which showed reactive lymphoid aggregates but ruled out lymphoma. Patient continued to deteriorate and died 1 month later due to massive pulmonary hemorrhage.

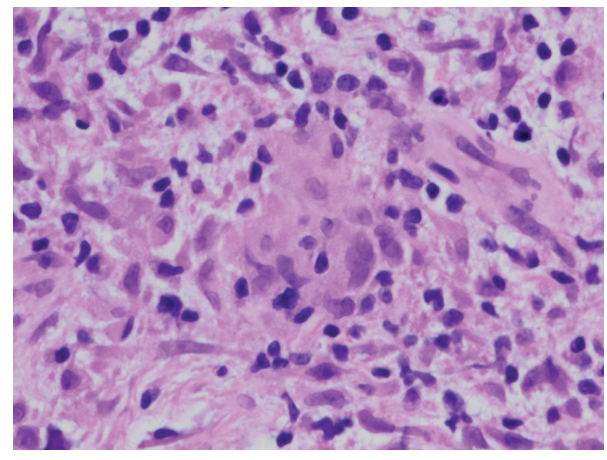

Figure 3: Photomicrograph of lacrimal gland biopsy shows a lympho-histiocytic infiltrate with reactive fibroblasts (hematoxylineosin, original magnification $\times 400)$.

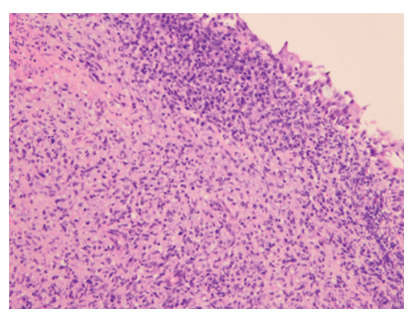

(a)

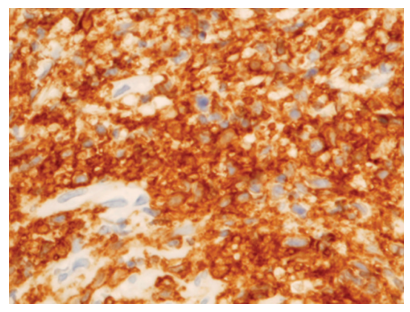

(c)

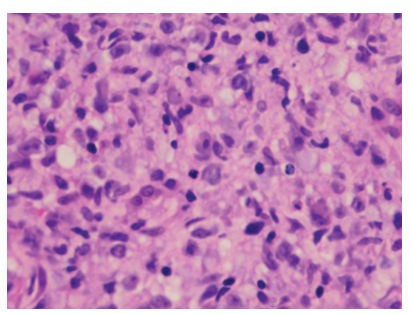

(b)

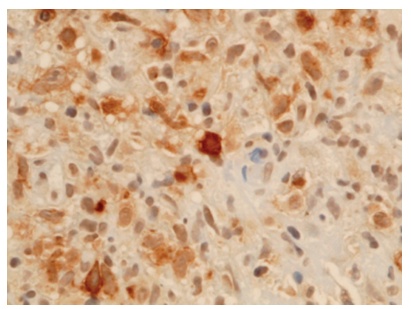

(d)
Figure 4: Transbronchial biopsy. (a) Photomicrograph of the transbronchial biopsy demonstrates submucosal lymphocytic inflammatory infiltrate (hematoxylin-eosin, original magnification $\times 100$ ). (b) Higher magnification shows a polymorphous lymphoid population with atypical lymphoid cells (hematoxylin-eosin, original magnifiaction $\times 400$ ). (c) Immunohistochemistry illustrates that the cells are predominantly B-cells (CD20 immunohistochemical stain, original magnification $\times 200$ ). (d) Immunohistochemistry for Epstein-Barr virus antigen demonstrates positive staining (EBVLMP immunohistochemical stain, original magnification $\times 400$ ).

\section{Discussion}

LG was first described by Leibow et al. [1] in 1972 as an atypical angiocentric and angiodestructive lymphoproliferative disease involving lungs as well as other extra nodal sites, mainly skin, CNS, and kidneys. In recent years it has been shown to be a T-cell rich EBV-related lymphoproliferative disease [17]. It can present at any age although $80 \%$ of the cases occur between 4 th and 6th decades. The incidence is higher in men with a male-tofemale ratio of $1.7: 1$ [3]. As with other EBV-associated lymphoproliferative disorders (such as post-transplant lymphoproliferative disorder), LG occurs with increased frequency 
in immunosuppressed patients. In its most indolent forms, it presents with pulmonary/skin nodules in an otherwise asymptomatic patient that resolve without any treatment. Fewer than $20 \%$ of the patients present with eye involvement, hepatosplenomegaly, adenopathy, and arthralgia [18]. Around $14-27 \%$ of the patients achieve durable remissions without treatment though a more likely course is that of relentless progression with multiple organ involvement and progression to large-cell lymphoma with a median survival of 14 months [3].

LG is characterized histologically by a polymorphic lymphoid infiltrate consisting of mature lymphocytes, eosinophils, histiocytes, and atypical lymphoreticular cells [1]. Focal areas of necrosis and transmural inflammation of vessels (angiitis) are also typical and extensive necrosis and monomorphic large-cell lymphoid infiltrate are indicative of a high-grade lesion. Since the histological findings are highly reminiscent of Wegener's granulomatosis, a much more common condition, the diagnosis of LG needs to be confirmed by demonstrating the presence of EBV positive B-cells within the lesion. A 3-tier grading system has been proposed based on the number of EBV positive cells [19]. The histological grading may vary over time or from site to site [18] and hence multiple site or repeated biopsies are essential to establish the diagnosis in cases with high index of clinical suspicion.

No well-studied effective treatment exists for this disease. Traditional treatment regimens were centered on immunosuppression (with cyclophosphamide and prednisolone) [5] but since the recognition that LGY is an EBV driven disease, antiviral therapy in the form of interferon-alpha is increasingly employed [18]. Combination chemotherapy is reserved for high-grade lesions. Newer therapies such as rituximab and autologous stem cell transplantation have also given good results [19-21]. Radiotherapy has also been used in certain cases [2], and proven to be an effective adjunct in treating localized disease.

The reports of ophthalmic involvement in LG [1-16, 22, 23] have been summarized in Table 1 . Twenty one cases were reviewed. Four cases were excluded because sufficient information was not available $[3,5]$. Excluding these cases, details of the remaining 17 cases were obtained and the age of onset, sex, ophthalmic presentation, the tissue biopsy used to establish diagnosis, treatment, and outcome were assessed.

The age at presentation ranged from 7 to 75 years (mean: 43.9 years) with equal incidence in both sexes: $(9$ male: 9 females). Of the 18 cases including our case, six presented with ocular symptoms while the remainder had pulmonary symptoms or central nervous involvement at initial diagnosis. The ophthalmic manifestations were due to infiltration of the ocular and orbital tissues in 12 cases; vasculitis caused functional disturbances in 3 cases; and ophthalmic expressions of the disease were seen in 4 cases from CNS disease. Eight of the 18 cases (44.4\%) presented acutely with progressive development of ocular manifestations within a month. The diagnosis was established in most cases by lung biopsy (13 cases). Only 4 cases had ophthalmic manifestations secondary to central nervous system (CNS) involvement (including diplopia, ptosis, nystagmus, and tonic pupil) contrary to earlier reports that ocular involvement in LG is commonly due to CNS involvement [23].

Five cases were treated with radiotherapy for the ocular manifestations. These included orbital infiltrations, eyelid swelling, and one case of conjunctival involvement, with all cases showing very good response with regression of symptoms. The remaining cases were treated with corticosteroids and/or immunosuppressants and in all cases the ocular manifestations resolved partially or completely except cases of optic neuropathy and optic nerve infarction. The follow up period for the cases ranged from 6 weeks to twelve years (mean $=3.15$ years). Of the 17 cases where follow up information was available, 8 succumbed to the disease with a median survival of 6 months (range: 6 weeks to 2 years).

In our case, the CT of the orbit revealed a nonhomogenous enlargement of lacrimal gland with central hypodense areas which corresponded with areas of necrosis within the lacrimal gland. This feature was of notable significance as the differential diagnoses included very few conditions such as necrotic or cystic changes within a pleomorphic or mucoepidermoid tumor of lacrimal gland and acute dacryoadenitis with abscesses within the gland [24-26].

Though ophthalmic manifestations are uncommon, they may rarely be the presenting feature in LG. Ophthalmologists should be aware of this condition, especially since many of the manifestations can mimic those of Wegener's granulomatosis. It is also a condition which may require multiple tissue biopsies to establish the diagnosis and one should not hesitate to do so in cases with high index of clinical suspicion. Given the systemic nature of the disease and poor prognosis of late-stage high-grade lesions, early diagnosis and appropriate treatment by an expert with an interest in this condition may offer the best hope for the patient.

\section{Acknowledgments}

Dr. Cannon received funding from the Royal College of Ophthalmologists, the Dickinson Trust, Pfizer, and the Ethicon Foundation for Ophthalmic Fellowship training in Adelaide. These sponsors had no role in the design or conduct of this study.

\section{References}

[1] A. A. Liebow, C. R. B. Carrington, and P. J. Friedman, "Lymphomatoid granulomatosis," Human Pathology, vol. 3, no. 4, pp. 457-558, 1972.

[2] B. B. Shank, C. D. Kelley, L. Z. Nisce, and D. Nori, "Radiation therapy in lymphomatoid granulomatosis," Cancer, vol. 42, no. 6, pp. 2572-2580, 1978.

[3] A. L. A. Katzenstein, C. B. Carrington, and A. A. Liebow, "Lymphomatoid granulomatosis: a clinicopathologic study of 152 cases," Cancer, vol. 43, no. 1, pp. 360-373, 1979.

[4] P. P. Sordillo, B. Epremian, B. Koziner, et al., "Lymphomatoid granulomatosis: an analysis of clinical and immunologic characteristics," Cancer, vol. 49, no. 10, pp. 2070-2076, 1982.

[5] A. S. Fauci, B. F. Haynes, J. Costa, et al., "Lymphomatoid granulomatosis. Prospective clinical and therapeutic 
experience over 10 years," The New England Journal of Medicine, vol. 306, no. 2, pp. 68-74, 1982.

[6] H. Saraux, M. Krulik, and L. Laroche, "Retinal arteritis during the course of Liebow's lymphomatoid granulomatosis," Journal Francais d'Ophtalmologie, vol. 6, no. 6-7, pp. 565-569, 1983.

[7] D. T. Tse, S. Mandelbaum, and D. A. Chuck, "Lymphomatoid granulomatosis with ocular involvement," Retina, vol. 5, no. 2, pp. 94-97, 1985.

[8] Y.-M. Chung, T.-S. Yeh, Y.-Y. Tsai, H. Chiang, and J.-H. Liu, "Conjunctival involvement in lymphomatoid granulomatosis," Ophthalmologica, vol. 196, no. 3, pp. 161-166, 1988.

[9] J. L. Kinyoun, R. E. Kalina, and M. L. Klein, "Choroidal involvement in systemic necrotizing vasculitis," Archives of Ophthalmology, vol. 105, no. 7, pp. 939-942, 1987.

[10] R. L. Font, P. S. Rosenbaum, and J. L. Smith Jr., "Lymphomatoid granulomatosis of eyelid and brow with progression to lymphoma," Journal of the American Academy of Dermatology, vol. 23, no. 2, pp. 334-337, 1990.

[11] D. McKay, J. Ell, R. Williams, and F. Taylor, "Lymphomatoid granulomatosis presenting as sudden blindness," Australian and New Zealand Journal of Ophthalmology, vol. 18, no. 2, pp. 215-219, 1990.

[12] A. D. J. Pearson, A. W. Craft, and J. M. Howe, "Choroidal involvement in lymphomatoid granulomatosis," British Journal of Ophthalmology, vol. 75, no. 11, pp. 688-689, 1991.

[13] S. Haider, "Tonic pupil in lymphomatoid granulomatosis," Journal of Clinical Neuro-Ophthalmology, vol. 13, no. 1, pp. 3839, 1993.

[14] S. Forman and P. S. Rosenbaum, "Lymphomatoid granulomatosis presenting as an isolated unilateral optic neuropathy. A clinicopathologic report," Journal of Neuro-Ophthalmology, vol. 18, no. 2, pp. 150-152, 1998.

[15] J. E. Head, D. Shen, M. Santiago-Maysonet, R. J. Bishop, and C.-C. Chan, "Ocular pathology of uncommon hematologic malignancies: a case series," Journal of Medical Case Reports, vol. 1, Article ID 158, 2007.

[16] J. R. Cameron and P. Cackett, "Lymphomatoid granulomatosis associated with bilateral exudative retinal detachments," Archives of Ophthalmology, vol. 125, no. 5, pp. 712-713, 2007.

[17] J. L. Myers, P. J. Kurtin, A.-L. A. Katzenstein et al., "Lymphomatoid granulomatosis: evidence of immunophenotypic diversity and relationship to Epstein-Barr virus infection," American Journal of Surgical Pathology, vol. 19, no. 11, pp. 1300-1312, 1995.

[18] E. S. Jaffe and W. H. Wilson, "Lymphomatoid granulomatosis: pathogenesis, pathology and clinical implications," Cancer Surveys, vol. 30, pp. 233-248, 1997.

[19] W. H. Wilson, D. W. Kingma, M. Raffeld, R. E. Wittes, and E. S. Jaffe, "Association of lymphomatoid granulomatosis with Epstein-Barr viral infection of B lymphocytes and response to interferon- $\alpha 2 b$," Blood, vol. 87, no. 11, pp. 4531-4537, 1996.

[20] K. Jordan, A. Grothey, W. Grothe, T. Kegel, H.-H. Wolf, and H.-J. Schmoll, "Successful treatment of mediastinal lymphomatoid granulomatosis with rituximab monotherapy," European Journal of Haematology, vol. 74, no. 3, pp. 263-266, 2005.

[21] A. Fassas, S. Jagannath, K. R. Desikan et al., "Lymphomatoid granulomatosis following autologous stem cell transplantation," Bone Marrow Transplantation, vol. 23, no. 1, pp. 79-81, 1999.

[22] C. L. Moertel, B. Carlson-Green, J. Watterson, and S. C. Simonton, "Lymphomatoid granulomatosis after childhood acute lymphoblastic leukemia: report of effective therapy," Pediatrics, vol. 107, no. 5, pp. E82-E90, 2001.

[23] J. B. Robin, D. J. Schanzlin, and D. M. Meisler, "Ocular involvement in the respiratory vasculitides," Survey of Ophthalmology, vol. 30, no. 2, pp. 127-140, 1985.

[24] J. A. C. L. Wharton and B. A. O'Donnell, "Unusual presentations of pleomorphic adenoma and adenoid cystic carcinoma of the lacrimal gland," Australian and New Zealand Journal of Ophthalmology, vol. 27, no. 2, pp. 145-148, 1999.

[25] W. S. Jung, K. J. Ahn, M. R. Park et al., "The radiological spectrum of orbital pathologies that involve the lacrimal gland and the lacrimal fossa," Korean Journal of Radiology, vol. 8, no. 4, pp. 336-342, 2007.

[26] N. Hosten, "Conditions involving the lacrimal gland and ducts," in Imaging of the Globe and Orbit. A Guide to Differential Diagnosis, pp. 110-116, Thieme, 1998. 


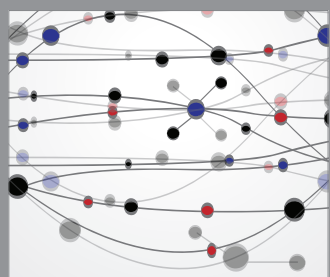

The Scientific World Journal
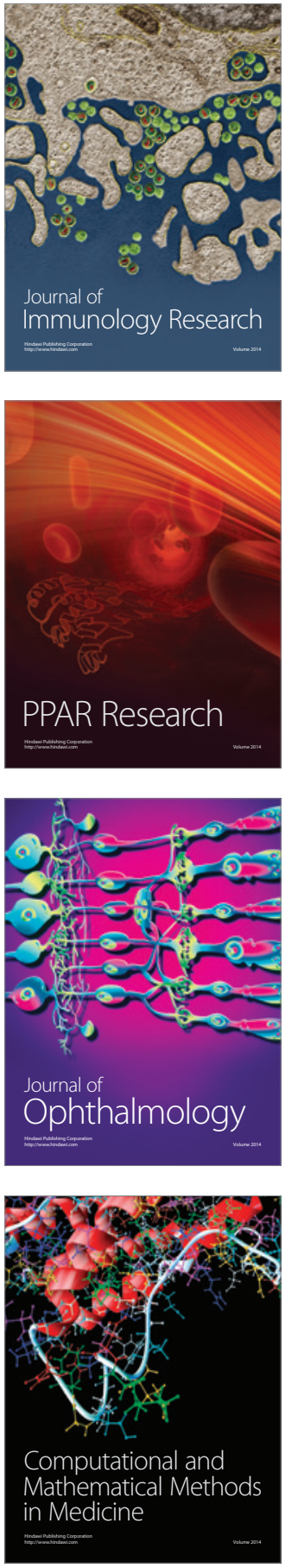

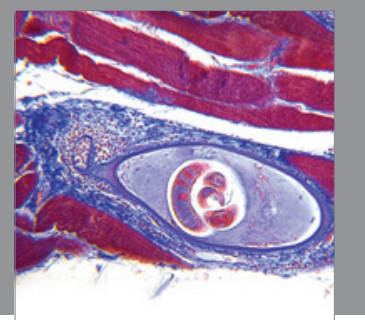

Gastroenterology

Research and Practice
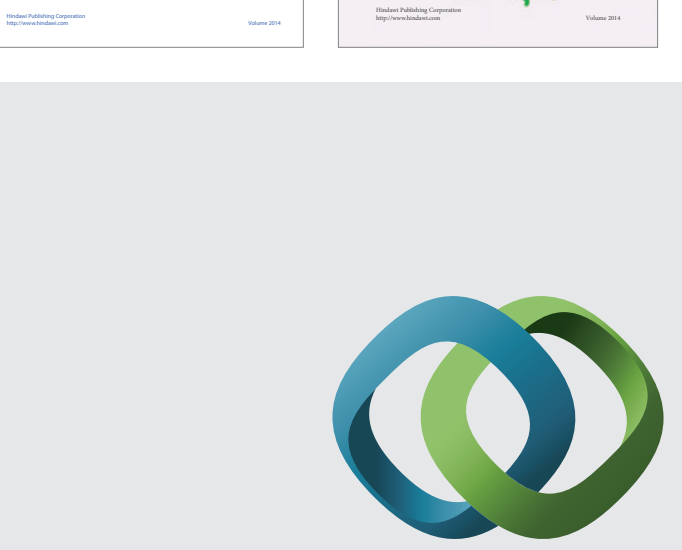

\section{Hindawi}

Submit your manuscripts at

http://www.hindawi.com
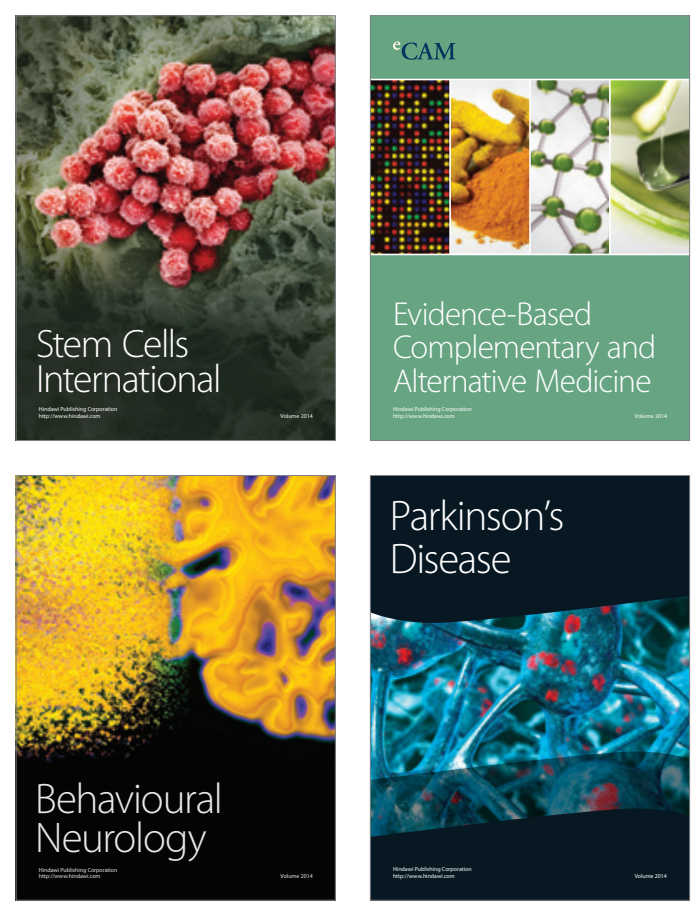

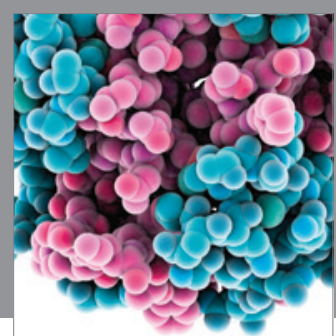

Journal of
Diabetes Research

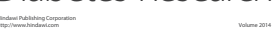

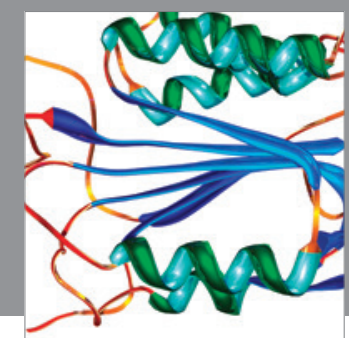

Disease Markers
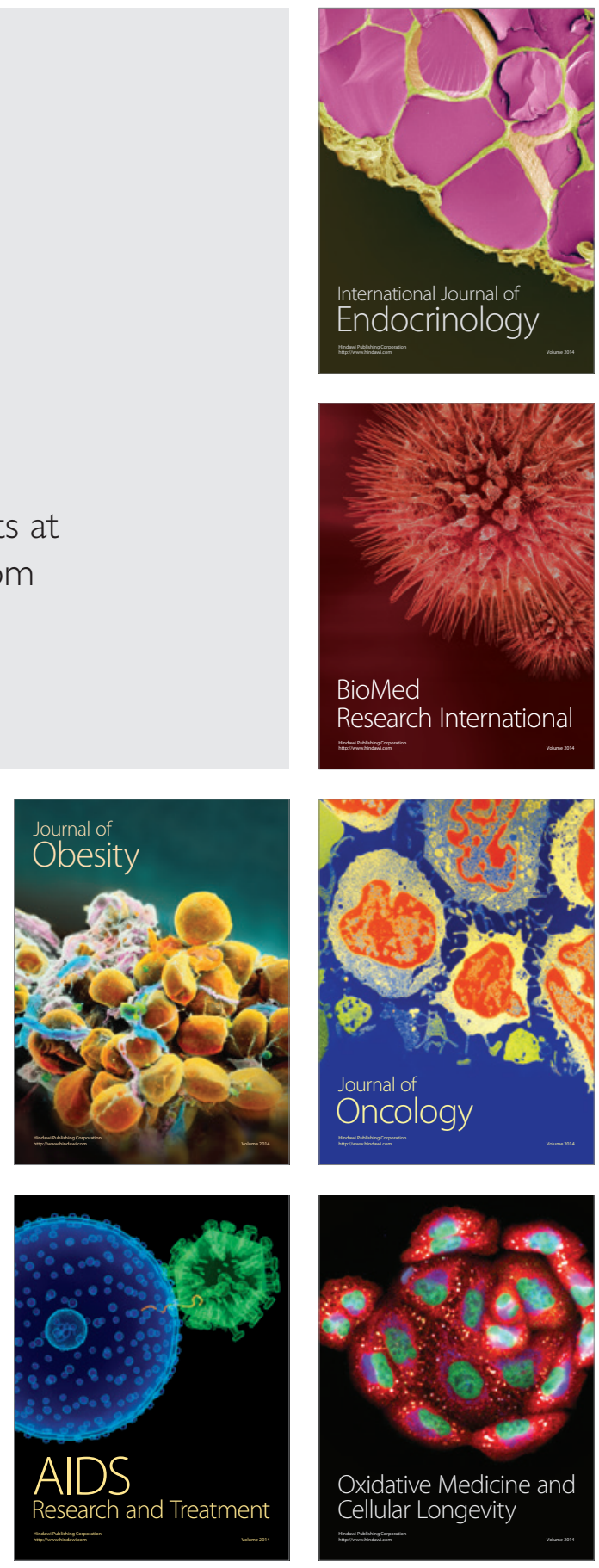